\title{
On the Feasibility of Integrating mmWave and IEEE 802.11p for V2V Communications
}

\author{
Marco Giordani $^{\circ}$, Andrea Zanella ${ }^{\circ}$, Takamasa Higuchi ${ }^{\dagger}$, Onur Altintas ${ }^{\dagger}$, Michele Zorzi ${ }^{\circ}$ \\ ${ }^{\circ}$ Consorzio Futuro in Ricerca (CFR) and University of Padova, Italy, Email:\{giordani,zanella,zorzi\}@ dei.unipd.it, \\ †TOYOTA InfoTechnology Center, Mountain View, CA, USA, Email:\{ta-higuchi,onur\}@us.toyota-itc.com
}

\begin{abstract}
Recently, the millimeter wave (mmWave) band has been investigated as a means to support the foreseen extreme data rate demands of emerging automotive applications, which go beyond the capabilities of existing technologies for vehicular communications. However, this potential is hindered by the severe isotropic path loss and the harsh propagation of high-frequency channels. Moreover, mmWave signals are typically directional, to benefit from beamforming gain, and require frequent realignment of the beams to maintain connectivity. These limitations are particularly challenging when considering vehicle-tovehicle (V2V) transmissions, because of the highly mobile nature of the vehicular scenarios, and pose new challenges for proper vehicular communication design. In this paper, we conduct simulations to compare the performance of IEEE 802.11p and the mmWave technology to support V2V networking, aiming at providing insights on how both technologies can complement each other to meet the requirements of future automotive services. The results show that mmWave-based strategies support ultra-high transmission speeds, and IEEE 802.11p systems have the ability to guarantee reliable and robust communications.
\end{abstract}

Index Terms-V2V communications; millimeter wave (mmWave); IEEE 802.11p; connectivity performance

\section{INTRODUCTION}

In recent years, Vehicle-to-Everything (V2X) communications have been investigated as a means to support automotive services to improve the efficiency and safety of road transportation systems. These safety services often deal with small data messages with very stringent requirements in terms of transmission reliability and latency [1]. However, future connected and automated vehicles will encompass a wide range of sensors, including video cameras, GPS, LIDARs and radars, that will generate massive amounts of data (e.g., in the order of terabytes per driving hour [2]) that may exceed the capacity of existing V2X communication technologies.

A possible response to this growing demand for ultrahigh transmission speeds can be found in the next-generation radio interfaces, globally standardized by the 3rd Generation Partnership Project (3GPP) as New Radio (NR) [3], that include in particular the millimeter wave (mmWave) bands. 1 Besides the extremely large bandwidths available at such frequencies, the small size of the antennas makes it possible to build complex antenna arrays and obtain high gains by beamforming (BF), thus further increasing the transmission

\footnotetext{
${ }^{1}$ Although strictly speaking mmWave bands include frequencies between 30 and $300 \mathrm{GHz}$, industry has loosely defined it to include any frequency above $10 \mathrm{GHz}$.
}

rates. The mmWave band therefore represents a new opportunity for future vehicular communications, in combination with other wireless systems. However, this potential can be jeopardized by the challenging propagation characteristics of high-frequency channels, as signals do not penetrate most solid materials and are subject to high signal attenuation and reflection. In addition, mmWave links are typically directional to benefit from beamforming gain and, especially when considering highly dense or highly mobile vehicular scenarios, require precise alignment of the transmitter and receiver beams to maintain connectivity [4], [5].

In this regard, while the research on Vehicle-toInfrastructure (V2I) systems operating at mmWaves has recently been quite widespread (e.g., [2], [8]-[11]), the literature on Vehicle-to-Vehicle (V2V) networking (e.g., [12], [13]) is still very scarce. In [12] the authors shed light on the operational limits of mmWave bands as a viable Radio Technology (RT) for future high-rate V2V transmissions while, in [13], a multi-access edge computing framework integrating licensed sub-6 $\mathrm{GHz}$ band and mmWaves for inter-vehicle information distribution is proposed. Some other papers have provided analytical characterizations of the mmWave channel in V2V scenarios. For example, in [14], the authors theoretically modeled the downlink performance of a mmWave network deployed along a highway section, while in [15] an experimental characterization of the $38 \mathrm{GHz}$ and $60 \mathrm{GHz}$ radio channel is presented. Motivated by these considerations, in this paper we conduct extensive simulations to provide the first numerical evaluation of the practical feasibility of designing mmWave-based strategies to target the requirements of future $\mathrm{V} 2 \mathrm{~V}$ services, and compare their performance to that of IEEE $802.11 \mathrm{p}$ systems. The results show that the support of highfrequency bands has the potential to provide extremely high data rates, while IEEE $802.11 \mathrm{p}$ enables reliable and robust communications with lower bit-rates. We conclude that the orchestration of different radios makes it possible to complement the limitations of each type of network and therefore represents a viable approach to improve the robustness and stability of $\mathrm{V} 2 \mathrm{~V}$ connectivity while establishing high-capacity channels.

The remainder of this paper is organized as follows. In Sec. П we overview the characteristics of the IEEE $802.11 \mathrm{p}$ standard and the mmWave technology in relation with target $\mathrm{V} 2 \mathrm{~V}$ application requirements. The system model is described in Sec. III] while in Sec. IV] we present our main findings and simulation results. Finally, conclusions and suggestions for future work are provided in Sec. V. 
TABLE I: Description of the characteristics of radio interfaces currently being considered for V2V communications.

\begin{tabular}{lll}
\hline RT & Pros & Cons \\
\hline IEEE 802.11p [6] & $\bullet$ No need for network infrastructure & $\bullet$ Hidden node problem \\
& $\bullet$ Low latency & $\bullet$ Limited transmission data rate \\
& $\bullet$ Fully distributed and uncoordinated access & \\
\hline mmWaves [7] & $\bullet$ Very large bandwidth & $\bullet$ Very large path loss \\
& $\bullet$ Beamforming gain & $\bullet$ Signals do not penetrate through solid material \\
& $\bullet$ Spatial isolation & $\bullet$ Seed to set up aligned transmissions \\
& $\bullet$ Potential of physical-layer security/privacy & Significant shadowing, reflection and scattering \\
\hline
\end{tabular}

\section{RAdio Technologies to EnABle V2V NETWORKING}

$\mathrm{V} 2 \mathrm{~V}$ communications are designed to exchange basic information among the vehicles to enable advanced automotive services, the main ones concerning the enhancement of road safety and the reduction of the traffic impact on the environment. The 3GPP has recently categorized different performance requirements for next-generation vehicular systems supporting enhanced V2V applications [16], as follows.

Vehicle platooning includes services that make it possible for a group of vehicles that follow the same trajectory to travel in close proximity to one another, nose-to-tail, at highway speeds. In addition to the strict latency requirement, the connection reliability and stability of these applications are also very critical.

Advanced driving enables semi- or fully-automated driving and allows vehicles to coordinate their trajectories and maneuvers to guarantee safer traveling, collision avoidance, and improved traffic efficiency. While the size of the exchanged safety messages is typically small (up to a few thousands of bytes), latency must be very small to ensure prompt reactions to unpredictable events.

The extended-sensor functionality enables the direct dissemination of raw or processed data, gathered through local sensors, among vehicles, which can enhance the perception of the surrounding environment beyond what their own instrumentation can detect. These services usually require highthroughput connections (in the order of hundreds of megabits per second), due to the detailed nature of the shared contents, while some latency can be tolerated (depending on the degree of automation).

It is therefore clear that future $\mathrm{V} 2 \mathrm{~V}$ services will have increasingly stringent demands in terms of data rate, reliability, latency, and connectivity. In this section, we thus overview the features of candidate RTs currently being considered for intervehicle communications, namely the IEEE 802.11p standard and the mmWave technology 2 and discuss their performance characteristics in relation with target application requirements. Table I provides a short summary of the argumentation.

\section{A. IEEE 802.11p Communications}

The IEEE 802.11p standard supports the Physical (PHY) and Medium Access Control (MAC) layers of the Dedicated

\footnotetext{
${ }^{2}$ Based on the 3 GPP Release 14 specifications, the cellular-V2X (C-V2X) technology, which relies on the PC5 interface specified for device-to-device operations, offers V2V services using cellular technologies as a basis [17. However, a comparison between C-V2X and the IEEE 802.11p and the mmWave paradigms is beyond the scope of this paper.
}

Short Range Communication (DSRC) transmission service and offers $\mathrm{V} 2 \mathrm{~V}$ data exchange at a nominal rate from 6 to 27 Mbps within a range of a few hundreds of meters [6]. In the US, DSRC can count on a total spectrum of $75 \mathrm{MHz}$ in the $5.9 \mathrm{GHz}$ frequency band, divided into seven $10-\mathrm{MHz}$ channels, with $5 \mathrm{MHz}$ of guard band at the lower end of the spectrum. Japan has been already deployed IEEE $802.11 \mathrm{p}$ systems using $10 \mathrm{MHz}$ of spectrum in the $760 \mathrm{MHz}$ band [18]. This standard embeds certain desirable features for $\mathrm{V} 2 \mathrm{~V}$ communications. Endpoints can operate without a network infrastructure, removing the need for prior exchange of control information and thus bringing a significant advantage in terms of latency with respect to regular Wi-Fi or legacy cellular operations. Moreover, IEEE 802.11p implements the carrier sensing multiple access with collision avoidance (CSMA/CA) mechanism at the MAC layer, thereby guaranteeing a fully distributed and uncoordinated access to the wireless channel, with no need for a resource allocation procedure.

Nevertheless, the IEEE 802.11p standard presents some inherent limitations. First, the throughput and delay performance degrades as the network load increases, even though there are ways of mitigating congestion by adjusting the message rate in the application layer [19]. Second, the channel access mechanism is prone to the hidden node problem, which may result in packet collisions. Third, the limited bandwidth of IEEE 802.11 p systems results in limited data rates which may not satisfy the requirements of some categories of future $\mathrm{V} 2 \mathrm{~V}$ applications, e.g., extended sensors.

In conclusion, on the one hand, IEEE 802.11p systems present desirable features in terms of ubiquitous connection availability and communication stability. On the other hand, some next-generation $\mathrm{V} 2 \mathrm{~V}$ use cases may require highthroughput transmissions beyond the capacity of existing DSRC systems.

\section{B. Millimeter Wave Communications}

The mmWave band between $10 \mathrm{GHz}$ and $300 \mathrm{GHz}$ has been considered as an enabler of the 5 th generation $(5 \mathrm{G})$ performance requirements in micro and picocellular networks [20]. As mentioned, these frequencies offer much more bandwidth than traditional networks operating in the congested bands below $6 \mathrm{GHz}$, and some preliminary capacity estimations have demonstrated that systems operating at mmWaves can offer orders of magnitude higher bitrates than legacy vehicular networks [21]. Moreover, the millimeter wavelengths make it practical to build very large antenna arrays (e.g., with 
32 or more elements) to provide spatial isolation, reduce interference, and support multiplexing.

Although this new band has gathered great interest for automotive applications, there are many concerns about its transmission characteristics [4]. First, with wavelengths in the order of millimeters, isotropic transmissions incur severe path loss and result in limited communication range. Second, unlike signals at sub-6 GHz frequencies, mmWave signals do not penetrate most solid materials, and movements of obstacles and reflectors may cause the channel to rapidly appear and disappear. Third, the significant Doppler spread experienced at high frequencies may impair the feedback over a broadcast channel (e.g., during synchronization or random access).

To overcome these limitations, contrary to legacy V2V schemes, mmWave communications are typically directional to benefit from beamforming gain, and support mechanisms by which the vehicles can quickly determine appropriate directions of transmission. This requires precise and continuous alignment of the transmitter and receiver beams to maintain connectivity, an operation that may increase the system overhead and lead to throughput degradation. In this regard, the definition of directional $\mathrm{V} 2 \mathrm{~V}$ strategies can be favored by the dissemination of in-vehicle sensors information, e.g., vehicle position information obtained from GPS measurements may help geometrically select the optimal beam to interconnect the endpoints at any given time and alleviate the burden of the beam alignment operations. However, the communication performance can be rather deteriorated if the data is inaccurate, outdated and/or unreliable. Moreover, albeit the implementation of a digital beamforming architecture allows the processing of multiple simultaneous and orthogonal beams in the digital domain and offers considerably faster alignment operations, it requires a separate Radio Frequency (RF) chain for each antenna element and therefore suffers from significant energy consumption and requires expensive hardware [22].

In conclusion, the design of $\mathrm{V} 2 \mathrm{~V}$ systems operating at mmWaves requires the implementation of mechanisms able to cope with the inherent instability of the high-frequency channels, a research challenge that is still largely unexplored.

\section{SYSTEM MODEL}

In this section we describe the system model we considered to evaluate the communication performance of a vehicular system implementing V2V transmissions. The path loss characterization is presented in Sec. III-A and Sec. III-B, while in $\mathrm{Sec}$. III-C we introduce our main simulation parameters.

\section{A. Non Line of Sight (NLOS) Probability}

In $\mathrm{V} 2 \mathrm{~V}$ systems, due to the relatively low elevation of the vehicle antennas, we reasonably expect that other vehicles will act as obstacles to the propagation of the signals. It is thus imperative to distinguish between Line of Sight (LOS) and Non Line of Sight (NLOS) path loss characterizations 3

\footnotetext{
${ }^{3}$ In order to better investigate the effect of dynamic blockages (i.e., vehicles) on the signal propagation, in our simulations we have not considered static obstacles, e.g., buildings or vegetation. A complete characterization of the NLOS path loss probability is however of great interest and is left for future work.
}

In this regard, in [23] the authors represent the blockage probability $P_{\mathrm{b}}(d)$, i.e., the probability of one or more vehicles potentially obstructing the LOS between the transmitter and the receiver, as a non-decreasing function of the inter-vehicle distance $d$ (the longer the link, the more likely to intersect with one or more blockages), i.e.,

$$
P_{\mathrm{b}}(d)=1-\min \left(1, \max \left(0, a d^{2}+b d+c\right)\right),
$$

where the parameters $a, b, c$ are derived from geometry-based deterministic simulations and depend on the vehicle density of the scenario. Nonetheless, from an electromagnetic perspective, the presence of intermediate vehicles potentially obstructing the visual line of sight between the endpoints does not necessarily imply the NLOS condition. It is also required that the Fresnel ellipsoid is not free of obstructions. Therefore, according to the analysis developed in [24], the probability $P_{\mathrm{NLOS}}(d)$ of NLOS condition for a link that spans a distance $d$ is given by the blockage probability multiplied by the probability that at least one vehicle is within the ellipsoid corresponding to $60 \%$ of the radius of the Fresnel zone, i.e.,

$$
P_{\mathrm{NLOS}}(d)=P_{\mathrm{b}}(d) \cdot Q\left(\frac{h-\mu_{h}}{\sigma_{h}}\right),
$$

where the Q-function $Q(\cdot)$ represents the tail distribution of the normal distribution function, and $\mu_{h}$ and $\sigma_{h}$ are the mean and the standard deviation of the obstacle height, respectively. Finally, $h$ denotes the effective height of the straight line connecting the transmitter and the receiver at the obstacle and is given by

$$
h=\left(h_{i}-h_{j}\right) \frac{d_{\mathrm{obs}}}{d}+h_{i}-0.6 r_{f}+\ell_{a},
$$

where $h_{i}$ and $h_{j}$ are the heights of the transmitting and the receiving vehicles, respectively, $d_{\text {obs }}$, which is uniformly distributed in $[0, d]$, is the distance between the transmitter and the obstacle, $\ell_{a}$ is the physical length of the antenna and $r_{f}$ is the radius of the first Fresnel ellipsoid, which is given by

$$
r_{f}=\sqrt{\frac{\lambda d_{\mathrm{obs}}\left(d-d_{\mathrm{obs}}\right)}{d}},
$$

with $\lambda$ denoting the wavelength.

\section{B. Path Loss Model}

As soon as the different communication states (i.e., LOS and NLOS) have been identified, the path loss follows a dualslope piecewise-linear model, which is deemed suitable to represent the real propagation in a vehicular environment. We distinguish between IEEE 802.11p and mmWave systems.

IEEE 802.11p Model. First, we define the term $\lfloor x\rceil_{a}^{b}$ as

$$
\lfloor x\rceil_{a}^{b} \triangleq \begin{cases}x, & \text { if } a \leq x \leq b \\ a, & \text { if } x<a \\ b, & \text { if } x>b\end{cases}
$$

Based on the above notation, the path loss for IEEE 802.11p systems follows the model in [25] and is expressed as

$$
\begin{aligned}
P L(d)= & \mathcal{A} \mathbb{1}_{N}+P L\left(d_{0}\right)-10 \gamma_{1} \log _{10}\left\lfloor\frac{d}{d_{0}}\right\rceil_{1}^{\frac{d_{c}}{d_{0}}}- \\
& -10 \gamma_{2} \log _{10}\left\lfloor\frac{d}{d_{c}}\right\rceil_{1}^{\infty}+\chi_{\sigma},
\end{aligned}
$$


where $\gamma_{1}$ and $\gamma_{2}$ are the path loss exponents, $\chi_{\sigma}$ represents the standard deviation of the shadowing, and $P L\left(d_{0}\right)$ is the free space path loss at the reference distance $d_{0}=1 \mathrm{~m}$. The critical parameter $d_{c}$ is the Fresnel distance and is calculated as

$$
d_{c}=\frac{4 h_{i} h_{j}}{\lambda}
$$

while the binary random variable $\mathbb{1}_{N}$ is equal to 1 with probability $P_{\mathrm{NLOS}}(d)$, i.e., in case of NLOS propagation. In such a situation, the path loss is increased by a factor $\mathcal{A}$ according to a knife-edge attenuation mode 4$]$ [24], i.e.,

$$
\mathcal{A}= \begin{cases}6.9+20 \log _{10}\left[\sqrt{(v-0.1)^{2}+1}+v-0.1\right], & \text { if } v>-0.7 \\ 0, & \text { otherwise }\end{cases}
$$

where $v=\sqrt{2} H / r_{f}$ and $H$ is the difference between the height of the obstacle and that of the line connecting the transmitting and the receiving vehicles.

Millimeter-Wave Model. Available measurements at mmWaves in the V2V context are very limited and realistic scenarios are indeed hard to simulate. In fact, the increased reflectivity and scattering from common objects and the poor diffraction and penetration capabilities of mmWaves are the main factors preventing the reuse of the existing sub- $6 \mathrm{GHz}$ path loss models for high-frequency scenarios [4]. Nevertheless, the authors in [26] have conducted some measurements at $60 \mathrm{GHz}$ to characterize the mmWave propagation between two cars communicating in LOS or NLOS situations. The path loss is calculated according to a dual-slope model, i.e.,

$$
P L(d)=\xi \cdot 10 \log _{10} d+\eta+15 \cdot d / 1000,
$$

where the rightmost term denotes the atmospheric attenuation at $60 \mathrm{GHz}$, that is $15 \mathrm{~dB} / \mathrm{km}$, and the parameters $\xi$ and $\eta$ are given in [26, Table VI]. In the case of LOS (with probability $\left.P_{\mathrm{LOS}}(d)=1-P_{\mathrm{NLOS}}(d)\right), \xi=1.77$ and $\eta=70$, while in the case of NLOS, as for our previous assumptions, we consider one single intermediate vehicle located midway between the transmitting and receiving vehicles, and we set $\xi=1.71$ and $\eta=78.6$.

\section{Simulation Parameters}

The simulation parameters are based on realistic system design considerations and are summarized in Table II Vehicles are deployed over a section of a highway, which is composed of $N_{l}=4$ infinitely long parallel lanes of width $w=3.5 \mathrm{~m}$, so that the total road width is $2 W=N_{l} \cdot w=14 \mathrm{~m}$. We consider a static scenario, therefore the impact of the vehicles' speed is not investigated at this early stage. Following the characterization proposed in the prior literature, the transmitting and receiving vehicles are modeled as sedan cars of height $h_{i}=h_{j}=142 \mathrm{~cm} \mathrm{[26]} \mathrm{while,} \mathrm{in} \mathrm{the} \mathrm{case} \mathrm{of} \mathrm{NLOS,} \mathrm{the}$ intermediate vehicle height is modeled as a normal random variable with mean $\mu_{h}=150 \mathrm{~cm}$ and standard deviation $\sigma_{h}=8.4 \mathrm{~cm}[24]$.

\footnotetext{
${ }^{4}$ For the tractability of the simulation, we assume that the attenuation factor $\mathcal{A}$ follows a single knife-edge model, which considers one single vehicle obstructing the LOS. The extension of the single knife-edge obstacle case

\begin{tabular}{|c|c|c|}
\hline Parameter & Value & Description \\
\hline $\begin{array}{l}w \\
N_{l} \\
P_{\mathrm{TX}} \\
h_{i}=h_{j} \\
\mu_{h} \\
\sigma_{h} \\
d\end{array}$ & $\begin{array}{l}3.5 \mathrm{~m} \\
4 \\
19.5 \mathrm{dBm} \\
142 \mathrm{~cm} \\
150 \mathrm{~cm} \\
8.4 \mathrm{~cm} \\
\{2, \ldots, 500\} \mathrm{m}\end{array}$ & $\begin{array}{l}\text { Lane width } \\
\text { Number of lanes } \\
\text { Transmission power } \\
\text { Height of TX and RX cars } \\
\text { Mean of intermediate car's height } \\
\text { Stddev. of intermediate car's height } \\
\text { Inter-vehicle distance }\end{array}$ \\
\hline $\begin{array}{l}W_{\mathrm{mmW}} \\
f_{\mathrm{c}, \mathrm{mmW}} \\
N\end{array}$ & $\begin{array}{l}400 \mathrm{MHz} \\
60 \mathrm{GHz} \\
\{4,64\}\end{array}$ & $\begin{array}{l}\text { mmWave total bandwidth } \\
\text { mmWave carrier frequency } \\
\text { Antenna array size }\end{array}$ \\
\hline $\begin{array}{l}W_{\mathrm{DSRC}} \\
f_{\mathrm{c}, \mathrm{DSRC}} \\
\ell_{a}\end{array}$ & $\begin{array}{l}75 \mathrm{MHz} \\
5.9 \mathrm{GHz} \\
10 \mathrm{~cm}\end{array}$ & $\begin{array}{l}\text { IEEE } 802.11 \mathrm{p} \text { total bandwidth } \\
\text { IEEE 802.11p carrier frequency } \\
\text { IEEE 802.11p antenna length }\end{array}$ \\
\hline \multicolumn{3}{|c|}{$\begin{array}{c}\gamma_{1}, \gamma_{2}, \xi_{1}, \chi_{2}(\text { IEEE } 802.11 \text { p path loss parameters }) \\
\xi, \eta(\text { mmWave path loss parameters }) \sim[26] \\
a, b, c \text { (blockage probability parameters }) \sim \mid 23]\end{array}$} \\
\hline
\end{tabular}
to a multiple knife-edge is not immediate and is left as future work.
}

TABLE II: Main simulation parameters.

IEEE 802.11 p systems operate in the legacy band, i.e., at $5.9 \mathrm{GHz}$, with a total bandwidth of $75 \mathrm{MHz}$. Antennas are supposed to be located on top of vehicles, in the middle of the roof (which was experimentally shown to be the overall optimum placement when considering omnidirectional transmissions [24]), and to be of length $\ell_{a}=10 \mathrm{~cm}$.

For mmWave links, the central frequency is set to $60 \mathrm{GHz}$ while the total bandwidth is set to $400 \mathrm{MHz}$, as specified in [3]. In order to establish directional transmissions, vehicles are equipped with Uniform Planar Arrays (UPAs) of $N$ elements, allowing to steer beams consisting of a main lobe of predefined width (which depends on $N$ ) and a side lobe that covers the remainder of the antenna radiation pattern 5 For completeness, in our study we also consider omnidirectional mmWave transmissions, i.e., $N=1$. For the beam alignment, we assume that measurement reports are periodically exchanged (i.e., at the beginning of every slot of duration $T$ ) among the vehicles so that they can periodically identify the optimal directions for their respective beams [5]. Such configuration is kept fixed for the whole slot, during which nodes may lose the alignment due to mobility. In case the connectivity is lost during a slot, it can only be recovered at the beginning of the subsequent slot, when the re-alignment procedure is performed again [5]. In this respect, geographical position data can be used to geometrically point the beam towards the intended receiver at any given time: the inaccuracy of such data is modeled according to a Gamma distribution with parameters $\alpha=3.14733$ and $\beta=0.462432$ [27].

Our results are derived through a Monte Carlo approach where $N_{\text {sim }}=100000$ independent simulations are repeated to get different statistical quantities of interest. In particular, we analyze the received signal strength between the transmitting and the receiving nodes for different values of the intervehicle distance $d$, with $d$ varying from $2 \mathrm{~m}$ to $500 \mathrm{~m}$. We recall that, with our channel model, the presence of the line of sight is probabilistically determined, thereby making the Monte Carlo approach a reasonable solution.

\footnotetext{
${ }^{5}$ It is quite reasonable to assume that four equal and independent antenna arrays are arranged on either side of each car and that the cars are positioned onto the same elevation plane, so that each array is responsible for covering a $\Delta_{\theta}=180^{\circ}$ azimuth space.
} 


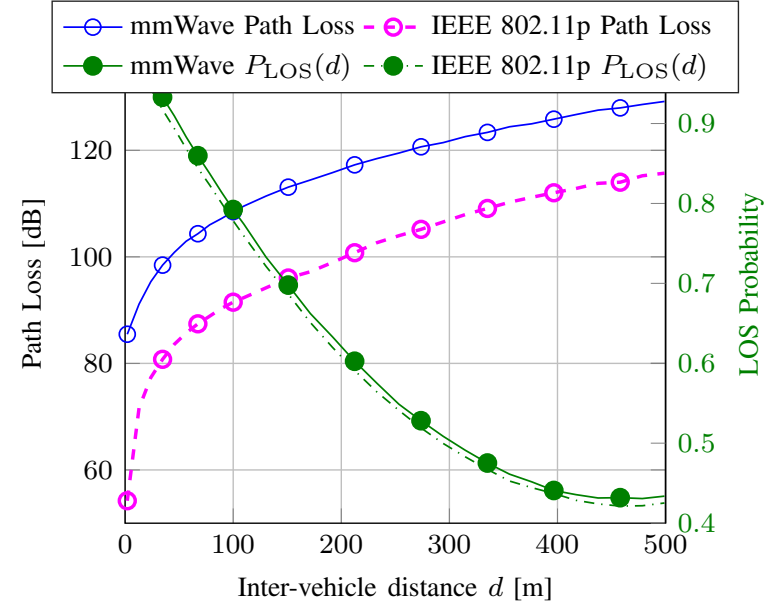

Fig. 1: Path loss (left axis) and LOS probability (right axis) $v s d$ for IEEE $802.11 \mathrm{p}$ and mmWave communications.

\section{Comparative Results}

In this section we provide some numerical results to compare the performance of IEEE 802.11p and mmWavebased $\mathrm{V} 2 \mathrm{~V}$ communications, which will be assessed in terms of achievable data rate, outage probability and stability.

Path Loss. Fig. 1 shows the path loss (left axis) and the LOS probability (right axis) as a function of the intervehicle distance $d$ and considering both IEEE 802.11p and mmWave transmissions. As expected, the LOS probability $P_{\mathrm{LOS}}(d)=1-P_{\mathrm{NLOS}}(d)$ is a non-increasing function of $d$ as the farther apart the vehicles, the more likely the event of obstruction of the line of sight. We notice that $P_{\mathrm{LOS}}(d)$ is slightly lower for IEEE $802.11 \mathrm{p}$ systems since, when operating at lower frequencies, the Fresnel radius $r_{f}$ increases, as highlighted by Eq. (4), thereby increasing the probability of vehicles obstructing the Fresnel ellipsoid. Nonetheless, the mmWave path loss is significantly higher than for IEEE $802.11 \mathrm{p}$ transmissions. The reason is that, unlike sub-6 $\mathrm{GHz}$ frequencies, mmWaves have increased reflectivity, poor diffraction and penetration capabilities in NLOS situations, and therefore are affected by significant attenuation. However, the effect of such properties is small for short distances (i.e., up to a few tens of meters), which therefore represent a suitable range for mmWave links in vehicular scenarios.

Data Rate. In Fig. 2, we compare the average Shannon data rate of both the IEEE $802.11 \mathrm{p}$ and the mmWave technologies, for different antenna configurations (including omnidirectional mmWave transmissions, i.e., $N=1)^{6}$ We observe that the very large bandwidth available to the mmWave systems (5 times larger than in IEEE 802.11p) ensures much higher throughput than operating at legacy frequencies (up to two

\footnotetext{
${ }^{6}$ In this paper, the Shannon data rate is computed from the Signal to Noise Ratio (SNR), which is the average received power divided by the noise power. Therefore, our results represent an upper bound for the performance of the vehicular nodes, as we do not investigate the effect of interference nor make any medium access control consideration.
}

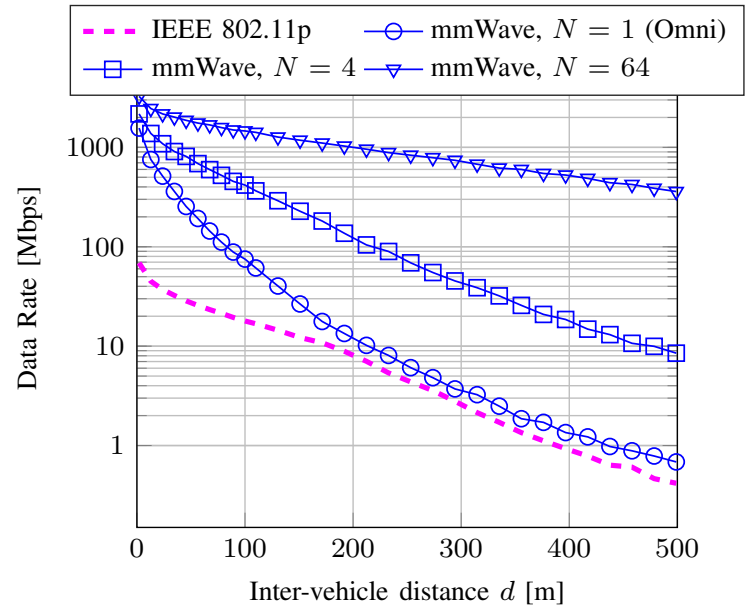

Fig. 2: Average achievable data rate $v s d$ for IEEE $802.11 \mathrm{p}$ (dashed line) and mmWave communications (solid lines), with different antenna array configurations (markers).

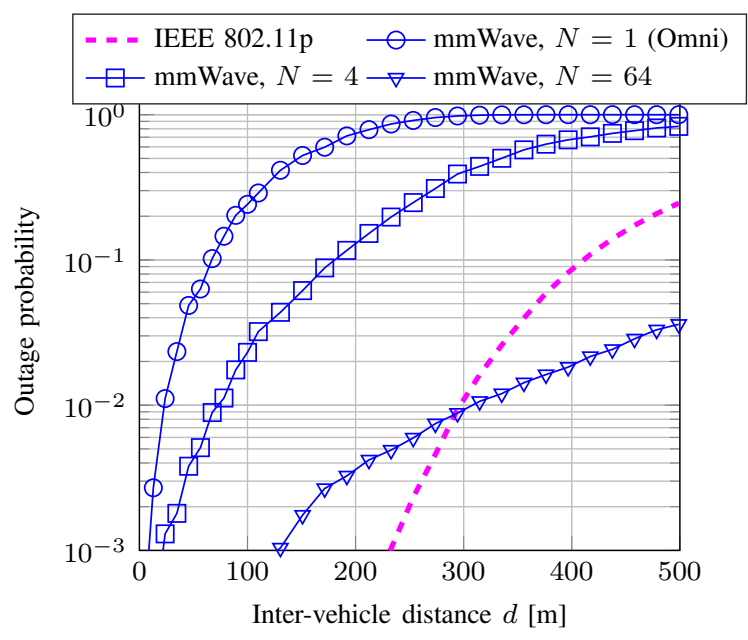

Fig. 3: Outage probability $v s d$ for IEEE $802.11 \mathrm{p}$ (dashed line) and mmWave communications (solid lines), with different antenna array configurations (markers).

orders of magnitude more in short range). This performance can be further magnified by configuring very directional transmissions. In fact, there exists a strong correlation among beamwidth, number of antenna elements and beamforming gain: the more antenna elements in the system, the narrower the beams, the more directional the transmission, the higher the gain that can be achieved by beamforming.

It should also be noted that, even implementing omnidirectional strategies at mmWaves, the connection still guarantees acceptable average bitrate, provided that the endpoints are sufficiently close (to increase the LOS probability).

Outage Probability. In Fig. 3, we evaluate the outage probability of the investigated $\mathrm{V} 2 \mathrm{~V}$ schemes, i.e., the probability that the received signal strength is below a predefined threshold, taken to be $-5 \mathrm{~dB}$ in our simulations. Low values of outage ensure more reliable $\mathrm{V} 2 \mathrm{~V}$ communications, a critical prerequisite for safety services requiring ubiquitous and continuous connectivity. 


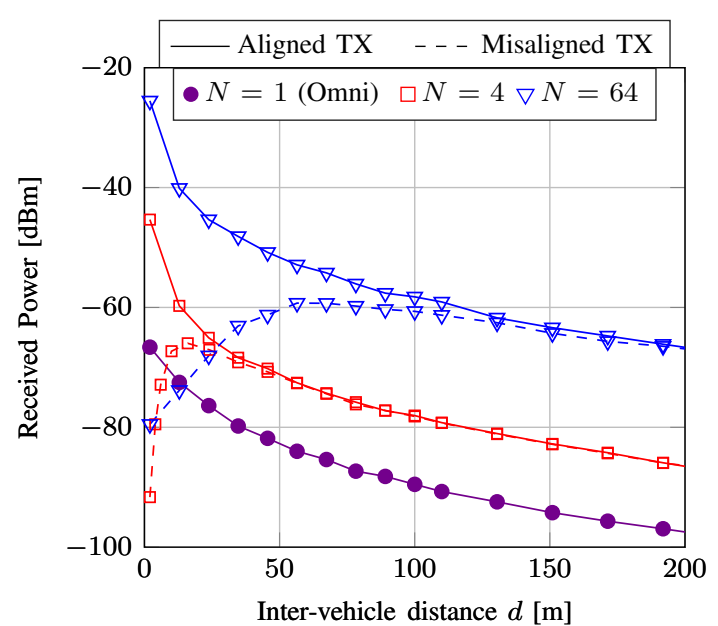

Fig. 4: Average received power $v s d$ for aligned (solid lines) and misaligned (dashed lines) mmWave communications, with different antenna array configurations (markers).

In general, we see that lower outage probability can be achieved when considering short-range communications and, in case of directional transmissions, when using large arrays. In the first case, the endpoints are progressively closer, thus ensuring better signal quality and stronger received power. In this region, the channel conditions are sufficiently good to ensure satisfactory signal quality (and, consequently, acceptable outage) even when considering small antenna factors or omnidirectional transmissions. In the second case, narrower beams are needed to guarantee higher gains, produced by beamforming.

Moreover, we observe that IEEE 802.11 p systems usually provide more reliable communications than mmWave links since they present a lower outage probability. Nevertheless, mmWave transmissions also achieve sufficient detection performance for spatially close vehicles (i.e., $d<110 \mathrm{~m}$ ) employing very narrow beams (e.g., $N=64$ ).

Finally, for very large distances (i.e., $d>300 \mathrm{~m}$ ), all the investigated configurations achieve unacceptable reliability values. However, mmWave communications with sharp beams (e.g., $N>64$ ) have the potential to support unreliable long-distance inter-vehicle communications for which the IEEE $802.11 \mathrm{p}$ signal is basically undetectable.

Robustness. The misalignment between the transmitter and the receiver, which can occur during lane change operations or as a consequence of the dissemination of inaccurate vehicle position information, may have a very detrimental impact on the performance of some $\mathrm{V} 2 \mathrm{~V}$ applications, as sensors may provide skewed or delayed readings and vehicles may lose connectivity. In our scenario, the degree $\delta_{m}$ of misalignment is distance-dependent and is given by $\delta_{m}=$ $\arctan (W / d)$, where $W=2 w=7 \mathrm{~m}$ is the width of one carriageway lane and $d$ is the inter-vehicle distance.

In Fig. 4 we observe that the impact of the misalignment on the communication performance depends on several factors, including $d$ and the beamwidth. In case of very directional mmWave transmissions (e.g., $N=64$ ), the quality of the

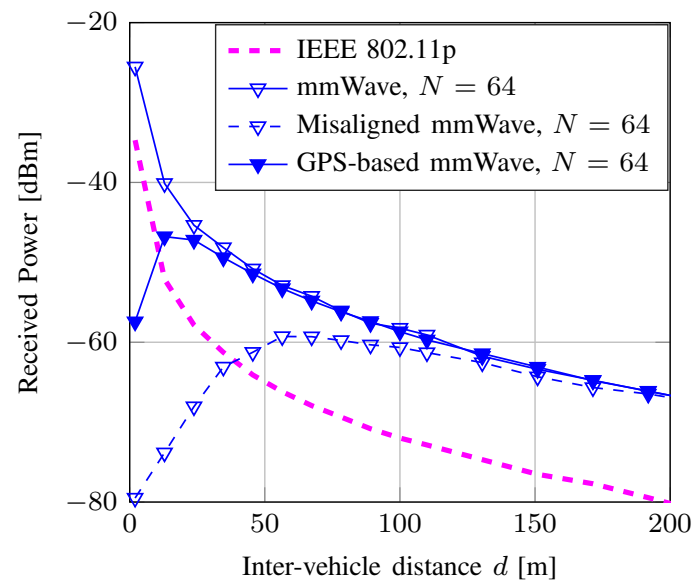

Fig. 5: Average received power $v s d$ for IEEE 802.11p (dashed line) and directional mmWave communications (solid lines), with $N=64$. If applicable, the inaccuracy of the geographical position data is modeled according to a Gamma distribution with parameters $\alpha=3.14733$ and $\beta=0.462432$ [27].

received signal significantly decreases as a result of misaligned nodes (i.e., more than $50 \mathrm{~dB}$ for short distances), mainly due to the non-continuous beamtracking mechanism: after the alignment is lost, vehicles need to wait for a new tracking operation to be performed to recover their optimal beam configuration. Conversely, more robust alignment can be achieved when considering smaller array factors since wider beams enlarge the area in which the vehicles are within coverage. Omnidirectional strategies are not affected by misalignment (in Fig. 4, the solid and dashed curves for the $N=1$ case overlap perfectly). In this approach, however, the well-known robustness versus throughput trade-off is exposed [5]: wider beams guarantee more robust and continuous connectivity but generally yield lower received power and transmission rates, as shown in Fig. 2 .

Moreover, from Fig. 4 we see that the impact of misalignment is dominant at short ranges. Indeed, the received power initially increases with $d$ since, for larger distances, the projection of the beam's shape onto the road surface is geometrically larger, thereby increasing the maximum distance that the vehicles can cover before leaving their respective communication ranges. However, beyond a certain value of $d$ (i.e., $d>50 \mathrm{~m}$ for $N=64$ and $d>10 \mathrm{~m}$ for $N=4$ ), beams are already sufficiently large to allow for loose alignment and the received power decreases just because of the path loss.

If sensory information (e.g., GPS coordinates) is available, it can be used to aid the configuration of the mmWave communication link and to remove the need for periodical beam tracking operations. In this regard, Fig. 5 reports the effect of misalignment due to inaccurate data, which makes the nodes point their beams towards improper directions. Nevertheless, such inaccuracy compromises only very shortrange transmissions (i.e., $d<20 \mathrm{~m}$ for $N=64$ ) 7

\footnotetext{
${ }^{7}$ The accuracy of the position information may be improved by the adoption of data fusion strategies which combine several localization techniques, e.g., dead reckoning, cellular localization, and camera image processing, into a single solution that is more robust and precise than any individual approach [28].
} 
Finally, Fig. 5 exemplifies how the omnidirectional transmissions of IEEE $802.11 \mathrm{p}$ systems offer more robust and, in some circumstances (e.g., $d<40$ ), more efficient V2V communications than their mmWave counterparts.

\section{Discussion AND Future Work}

In this paper we provided the first numerical comparison of the performance, in terms of achievable data rate, detection accuracy and robustness, between IEEE $802.11 \mathrm{p}$ (the current standard for short-range vehicular communications) and the mmWave technology to support V2V networking. Overall, we showed that IEEE $802.11 \mathrm{p}$ systems offer low-rate connectivity (i.e., up to a few tens of Mbps) but guarantee very stable, reliable and robust transmissions at short/medium distances (i.e., up to a few hundreds of meters) thanks to the intrinsic stability of the low-frequency channels and the omnidirectional transmissions. Conversely, mmWave systems support very high-throughput connections but exhibit high instability due to the severe signal propagation characteristics and the need to maintain beam alignment. Although the connectivity robustness can be magnified by considering transmissions through wide beams, the data rate increases considering very directional communications and close-range vehicles instead. We also showed that sensory information has the potential to help reducing the beam alignment overhead with minor performance degradation even in the presence of inaccurate data.

In this context, we conclude that the synergistic orchestration among the different radios makes it possible to complement the limitations of each type of network and deliver more flexible and resilient transmissions.

This work opens up some particularly interesting research directions, such as the definition of interface selection mechanisms able to dynamically identify the recommended type of radio to interconnect the vehicles. Moreover, due to the lack of temporally and spatially correlated channel measurements in the mmWave band, it is currently not possible to accurately evaluate the performance of the vehicles in realistic mobilityrelated scenarios, which on the other hand remains a very interesting and relevant item for future research.

\section{REFERENCES}

[1] H. Hartenstein and K. Laberteaux, VANET vehicular applications and inter-networking technologies. John Wiley \& Sons, 2009.

[2] J. Choi, V. Va, N. Gonzalez-Prelcic, R. Daniels, C. R. Bhat, and R. W. Heath, "Millimeter-Wave Vehicular Communication to Support Massive Automotive Sensing," IEEE Communications Magazine, vol. 54, no. 12, pp. 160-167, December 2016.

[3] 3GPP, "Study on New Radio (NR) Access Technology - Physical Layer Aspects (Release 14)," TS 38.802, 2017.

[4] M. Giordani, A. Zanella, and M. Zorzi, "Millimeter wave communication in vehicular networks: Challenges and opportunities," in 6th International Conference on Modern Circuits and Systems Technologies (MOCAST), May 2017.

[5] M. Giordani, M. Rebato, A. Zanella, and M. Zorzi, "Coverage and Connectivity Analysis of Millimeter Wave Vehicular Networks," submitted to Ad Hoc Networks, 2018. [Online]. Available: https://arxiv.org/abs/1803.01136

[6] Y. J. Li, "An overview of the DSRC/WAVE technology," in International Conference on Heterogeneous Networking for Quality, Reliability, Security and Robustness. Springer, 2010, pp. 544-558.

[7] T. S. Rappaport, R. W. Heath Jr, R. C. Daniels, and J. N. Murdock, Millimeter wave wireless communications. Pearson Education, 2014.
[8] V. Va, T. Shimizu, G. Bansal, and R. W. Heath, "Beam design for beam switching based millimeter wave vehicle-to-infrastructure communications," in IEEE International Conference on Communications (ICC), May 2016.

[9] N. Garcia, H. Wymeersch, E. G. Strom, and D. Slock, "Location-aided mm-wave channel estimation for vehicular communication," in IEEE 17th International Workshop on Signal Processing Advances in Wireless Communications (SPAWC), July 2016.

[10] N. González-Prelcic, R. Méndez-Rial, and R. W. Heath Jr, "Radar aided beam alignment in mmwave V2I communications supporting antenna diversity," in Proceedings of the 2016 Information Theory and Applications Workshop, 2016.

[11] M. Giordani, A. Zanella, T. Higuchi, O. Altintas, and M. Zorzi, "Performance Study of LTE and mmWave in Vehicle-to-Network Communications," IEEE 17th Annual Mediterranean Ad Hoc Networking Workshop (Med-Hoc-Net), June 2018.

[12] C. Perfecto, J. D. Ser, and M. Bennis, "Millimeter-Wave V2V Communications: Distributed Association and Beam Alignment," IEEE Journal on Selected Areas in Communications, vol. 35, no. 9, pp. 2148-2162, Sept 2017.

[13] Q. Hu, C. Wu, X. Zhao, X. Chen, Y. Ji, and T. Yoshinaga, "Vehicular Multi-Access Edge Computing With Licensed Sub-6 GHz, IEEE 802.11p and mmWave," IEEE Access, vol. 6, pp. 1995-2004, 2018.

[14] A. Tassi, M. Egan, R. J. Piechocki, and A. Nix, "Modeling and design of millimeter-wave networks for highway vehicular communication," IEEE Transactions on Vehicular Technology, vol. 66, no. 12, pp. $10676-$ 10691, Dec 2017

[15] M. G. Sánchez, M. P. Táboas, and E. L. Cid, "Millimeter wave radio channel characterization for 5G vehicle-to-vehicle communications," Measurement, vol. 95, pp. 223-229, 2017.

[16] 3GPP, "Technical Specification Group Services and System Aspects; Enhancement of 3GPP support for V2X scenarios; Stage 1 (Release 15)," TS 22.186, 2017.

[17] —, "Study on architecture enhancements for LTE support of V2X services (Release 14)," TS 23.785, 2016.

[18] Toyota Motor Corporation, "Toyota bringing advanced its technology to mass-market models," September 2015. [Online]. Available: http://newsroom.toyota.co.jp/en/detail/9676551

[19] G. Bansal, J. B. Kenney, and C. E. Rohrs, "Limeric: A linear adaptive message rate algorithm for dsrc congestion control," IEEE Transactions on Vehicular Technology, vol. 62, no. 9, pp. 4182-4197, Nov 2013.

[20] S. Rangan, T. S. Rappaport, and E. Erkip, "Millimeter-wave cellular wireless networks: Potentials and challenges," Proceedings of the IEEE, vol. 102, no. 3, pp. 366-385, March 2014.

[21] A. Kato, K. Sato, and M. Fujise, "ITS wireless transmission technology. Technologies of millimeter-wave inter-vehicle communications: Propagation characteristics," Journal of the Communications Research Laboratory, vol. 48, pp. 99-110, March 2001.

[22] W. B. Abbas and M. Zorzi, "Towards an appropriate receiver beamforming scheme for millimeter wave communication: A power consumption based comparison," in 22th European Wireless Conference, May 2016.

[23] M. Boban, X. Gong, and W. Xu, "Modeling the Evolution of Line-ofSight Blockage for V2V Channels," in IEEE 84th Vehicular Technology Conference (VTC-Fall), Sept 2016.

[24] M. Boban, T. T. V. Vinhoza, M. Ferreira, J. Barros, and O. K. Tonguz, "Impact of Vehicles as Obstacles in Vehicular Ad Hoc Networks," IEEE Journal on Selected Areas in Communications, vol. 29, no. 1, pp. 15-28, January 2011.

[25] L. Cheng, B. E. Henty, D. D. Stancil, F. Bai, and P. Mudalige, "Mobile Vehicle-to-Vehicle Narrow-Band Channel Measurement and Characterization of the $5.9 \mathrm{GHz}$ Dedicated Short Range Communication (DSRC) Frequency Band," IEEE Journal on Selected Areas in Communications, vol. 25 , no. 8, pp. 1501-1516, Oct 2007.

[26] A. Yamamoto, K. Ogawa, T. Horimatsu, A. Kato, and M. Fujise, "Pathloss prediction models for intervehicle communication at $60 \mathrm{GHz}$," IEEE Transactions on Vehicular Technology, vol. 57, no. 1, pp. 65-78, Jan 2008.

[27] T. Driver, "Long-term prediction of GPS accuracy: Understanding the fundamentals," in Proceedings of the 20th International Technical Meeting of the Satellite Division of The Institute of Navigation (ION GNSS), 2007.

[28] N. Alam, A. T. Balaei, and A. G. Dempster, "Relative positioning enhancement in VANETs: A tight integration approach," IEEE Transactions on Intelligent Transportation Systems, vol. 14, no. 1, pp. 47-55, June 2013. 